\title{
铜催化的不对称叠氮和炔烃的环加成反应的研究进展
}

\author{
王 才 ${ }^{a}$ 周 锋*,a 周 剑*,a,b \\ ( ${ }^{a}$ 华东师范大学化学与分子工程学院 绿色化学与化工过程绿色化上海市重点实验室 上海 200062) \\ ( ${ }^{b}$ 中国科学院上海有机化学研究所 金属有机化学国家重点实验室 上海 200032)
}

\begin{abstract}
摘要 尽管铜催化的叠氮和炔烃环加成 $(\mathrm{CuAAC})$ 反应作为点击化学的核心反应得到广泛应用, 然而其不对称催化的研 究却相对滞后. 反应本身虽然不产生手性元素，但是能够通过对具有潜手性的炔烃或叠氮化合物的去对称化以及外消 旋叠氮或炔烃化合物的动力学拆分, 来不对称催化合成手性叠氮、炔烃和三氮唑类具有重要应用价值的化合物. 自 2013 年首例高对映选择性的 CuAAC 反应被报道以来，相关研究有了重要进展，并被成功用于中心手性、轴手性和平 面手性的构建. 概述了不对称 CuAAC 反应的研究进展, 讨论其面临的发展难题和未来的发展空间.

关键词 铜催化的叠氮和炔烃环加成(CuAAC)反应; 去对称化; (动态)动力学拆分
\end{abstract}

\section{Recent Advances in the Enantioselective Copper(I)-Catalyzed Azide-Alkyne Cycloaddition Reaction}

\author{
Wang, $\mathrm{Cai}^{a} \quad$ Zhou, Feng ${ }^{*, a} \quad$ Zhou, Jian ${ }^{*, a, b}$ \\ ( ${ }^{a}$ Shanghai Key Laboratory of Green Chemistry and Chemical Processes, School of Chemistry and \\ Molecular Engineering, East China Normal University, Shanghai 200062) \\ ( ${ }^{b}$ State Key Laboratory of Organometallic Chemistry, Shanghai Institute of Organic Chemistry, \\ Chinese Academy of Sciences, Shanghai 200032)
}

\begin{abstract}
As one of the most important click reactions, the copper-catalyzed azide-alkyne cycloaddition (CuAAC) has found widespread applications. Nevertheless, the exploitation of enantioselective CuAAC remains largely undeveloped. Although the reaction itself does not generate chiral elements, the enantioselective CuAAC can be realized via the desymmetrization of prochiral dialkynes or diazides, as well as the kinetic resolution of racemic azides or terminal alkynes. Notably, enantioselective CuAAC provides efficient access to structurally diverse enantioenriched compounds featuring an azide, terminal alkyne or 1,4-disubstituted 1,2,3-triazoles, which are valuable structural units in organic synthesis or medicinal chemistry. Since the first highly enantioselective CuAAC reaction via desymmetrization of prochiral diynes was reported in 2013, substantial progress has been made in this research area. To date, enantioselective CuAAC has been successfully applied to the construction of central chirality, axial chirality and planar chirality. The recent exciting achievements are summarized, the challenges in this context are briefly discussed, and the synthetic opportunities for future development are outlined.

Keywords copper-catalyzed azide-alkyne cycloaddition (CuAAC) reaction; desymmetrization; (dynamic) kinetic resolution
\end{abstract}

1963 年, Huisgen ${ }^{[1]}$ 对高温条件下叠氮与炔烃的 $1,3-$ 偶极环加成反应进行了研究, 然而由于反应条件苛刻且 区域选择性不理想，很大程度上限制了该反应的应用. 2002 年, Meldal 和 Sharpless 小组 ${ }^{[2]}$ 分别独立报道了铜催 化的叠氮与炔烃的环加成反应(简称 $\mathrm{CuAAC}$ ). 他们发 现, 使用一价铜作为催化剂, 能够单一地得到 1,4-取代 的 1,2,3-三氮唑类化合物. 由于其具有区域选择性高、
底物普适性广、反应效率高且条件温和等优点, 符合 2001 年 Sharpless 等 ${ }^{[3]}$ 提出的 “点击化学” 的概念. 至此, CuAAC 反应得到了广泛研究, 并作为点击化学的精华 在生物化学、超分子化学和材料化学等研究领域得到了 广泛应用 ${ }^{[4]}$.

CuAAC 反应的机理研究因此得到了关注, 早期研 究认为可能是单核铜催化的过程 ${ }^{[5]}$, 然而随后的理论计

\footnotetext{
* Corresponding authors. E-mail: fzhou@chem.ecnu.edu.cn; jzhou@chem.ecnu.edu.cn Received May 9, 2020; revised May 28, 2020; published online June 8, 2020. Dedicated to Professor Henry N. C. Wong on the occasion of his 70th birthday. Project supported by the National Natural Science Foundation of China (Nos. 21672068, 21871090) 国家自然科学基金(Nos. 21672068, 21871090)资助项目.
} 
算和动力学实验表明, 反应可能涉及多核铜络合物中间 体. 2013 年, Fokin 等 ${ }^{[5 b]}$ 基于同位素交叉等实验，提出了 双铜协同催化的机理, 认为两个铜原子在活化炔烃和叠 氮的同时, 即时组织两者以类似于分子内反应的方式进 行反应，从而高选择地得到 1,4-三氮唑产物(Scheme 1).

尽管 $\mathrm{CuAAC}$ 反应本身不产生手性, 但是通过对具 有潜手性的炔烃或叠氮的去对称化以及外消旋叠氮或 炔烃化合物的(动态)动力学拆分, 均可以发展不对称催 化的 CuAAC 反应(Scheme 2), 进而实现含手性的三氮 唑化合物的高效合成. 由于该类结构单元具有和肽键类 似的电子特性和结构特点, 同时不易水解, 是一类重要 的药效基团, 在药物和生物活性分子中普遍存在 ${ }^{[6]}$; 此 外, 通过动力学拆分还能够为具有叠氮或者末端炔烃官 能团的手性化合物提供新的不对称催化合成方法, 具有 上述两种官能团的化合物均是重要的手性合成子, 能够 发生多种转化，然而其合成方法并不多 ${ }^{[7]}$.

尽管不对称 CUAAC 反应可用于合成含三氮唑和具 有末端炔基或叠氮基的手性化合物, 但是其研究相较于 $\mathrm{CuAAC}$ 反应在众多领域的成功应用十分滞后. 直到 2005 年, Fokin 和 Finn 等 ${ }^{[8]}$ 才利用去对称化和动力学拆 分两种策略进行了尝试, 但是结果均不理想, 基于外消 旋叠氮的动力学拆分仅能取得 8 的拆分因子 ( $s$-factor)
(Scheme 3a). 令人吃惊的是基于偕二叠氮的去对称化反 应仅能取得中等对映选择性，且基本以双三氮唑副产物 为主(Scheme 3b). 他们对此现象做了进一步研究 ${ }^{[9]}$, 发 现在无配体条件下，双叠氮参与的 CuAAC 反应，尽管 使用 10 equiv. 叠氮 8 与炔烃 $\mathbf{2}$ 反应, 仍然是双三氮唑产 物 10 为主, 仅得到痕量单三氮唑 9 (Scheme 4). 他们认 为三氮唑铜络合物 I 能进一步与炔烃作用(II 或 III), 使 其易与分子中的另一叠氮发生反应. 此外, 单三氮唑化 合物 9 也可能进一步发生反应得到双三氮唑化合物. 双 炔体系的反应同样是得到双三氮唑为主的产物. 这一发 现, 充分说明发展去对称化的 CuAAC 反应十分困难.

上述早期探索说明发展不对称 CuAAC 反应具有很 高的挑战性, 从而导致随后 8 年这一研究陷入沉寂, 未 见任何报道. 由于 $\mathrm{CuAAC}$ 反应可能经历双核铜催化机 理, 且末端炔烃和叠氮均为线型结构, 因此其与手性铜 络合物的识别模式，较常见的醛、酮、亚胺等单齿或双 齿配位型底物有所不同. 此外, 无论是通过去对称化还 是动力学拆分发展不对称 $\mathrm{CuAAC}$ 反应，底物的潜手性 中心或已有的手性中心, 均与反应位点距离较远, 而通 过 “远程控制” 来实现理想的立体选择性对手性催化剂 要求很高. 其次, 反应过程中可能存在的一系列副反应 同样限制了不对称 CuAAC 反应的发展. 由 Scheme 1 所

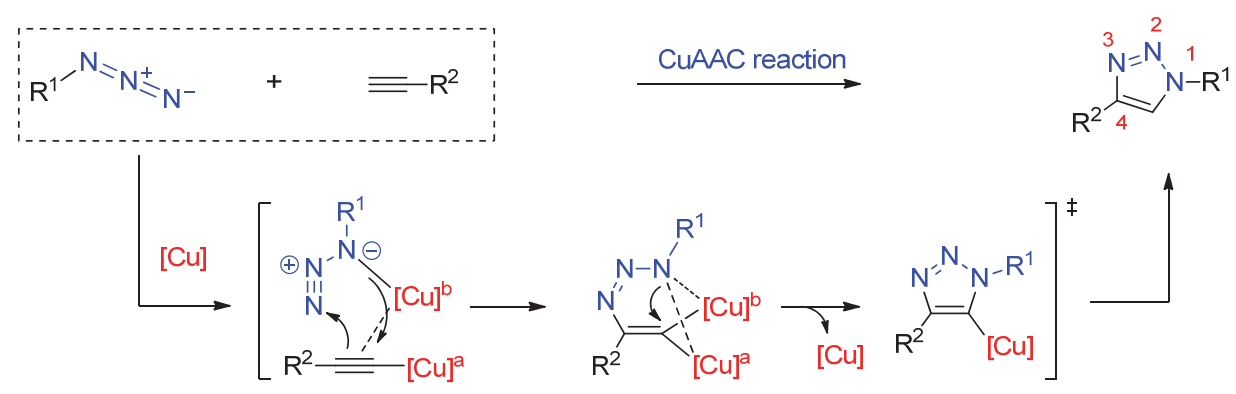

图式 $1 \mathrm{CuAAC}$ 反应及其可能机理

Scheme 1 CuAAC reaction and its possible mechanism

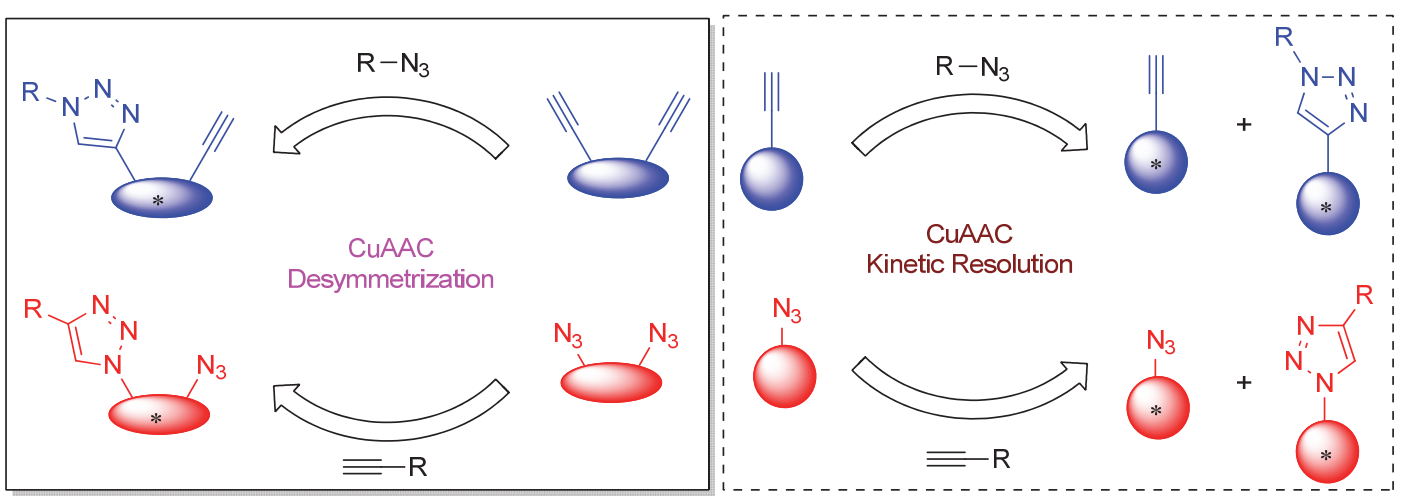

图式 2 不对称 $\mathrm{CuAAC}$ 反应的两种策略

Scheme 2 Two strategies of asymmetric CuAAC 
(a)

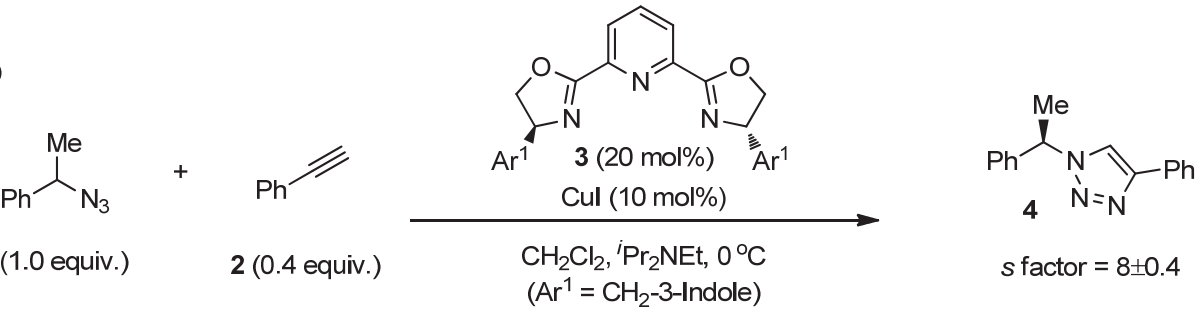

(b)<smiles>C#C[PH](=O)O[Mg]</smiles>

Cul (10 mol\%), 3 (20 mol\%) $\mathrm{CH}_{2} \mathrm{Cl}_{2}$, r.t. $\left(\mathrm{Ar}=4-\mathrm{ClC}_{6} \mathrm{H}_{4}\right)$

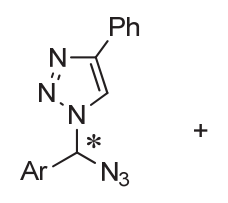

6, $25 \%, 59 \%$ ee

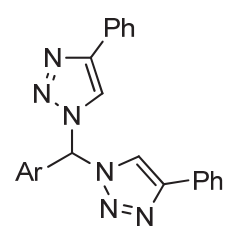

$7,63 \%$

图式 3 首例不对称 $\mathrm{CuAAC}$ 反应

Scheme 3 The first research of asymmetric CuAAC reaction

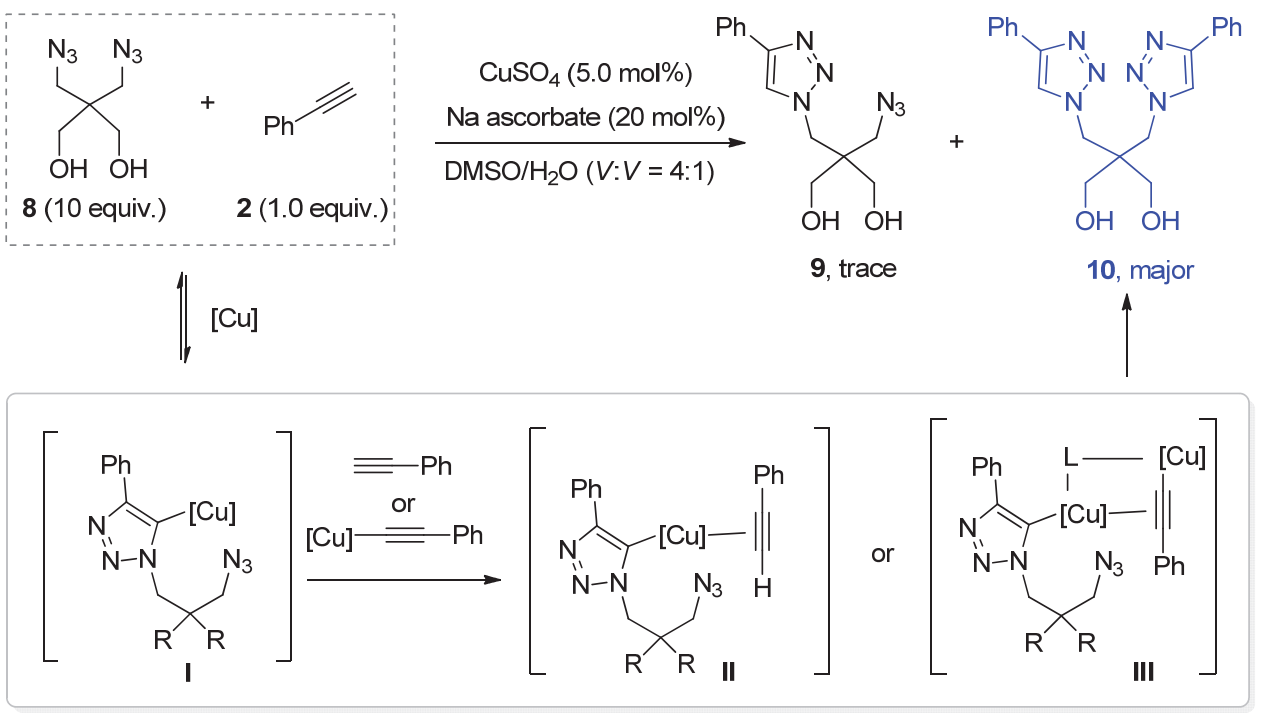

图式 4 无配体参与的 $\mathrm{CuAAC}$ 反应

Scheme 4 CuAAC reaction under ligand free condition

示机理可知, 反应经历炔基铜和三氮唑铜中间体, 而手 性配体的引入引起的手性铜催化剂的空间环境和电性 的改变, 有可能导致诸如 Glaser 偶联副反应的发生; 此 外, 发展去对称化 CuAAC 反应还需要在取得优异选择 性的时候，抑制非手性的双三氮唑生成. 这些是发展高 选择性的不对称 CuAAC 反应必须克服的问题.

2013 年, 周剑小组 ${ }^{[10]}$ 实现了首例高对映选择性的 不对称 CuAAC 反应. 至此, 该研究重新引起了化学家 的关注，近年来逐渐实现了一系列含三氮唑、炔基或叠 氮基团的碳或磷中心手性、轴手性以及平面手性化合物 的合成. 尽管 Fossey 等 ${ }^{[11]}$ 在 2016 年对不对称 CuAAC 反应做了亮点介绍, 但是迄今已有四年, 不能反映最新 进展. 因此, 本文将对该领域的研究进行全面的综述, 按照去对称化的和动力学拆分的 $\mathrm{CuAAC}$ 反应进行分类,
而前者进一步按手性中心、手性轴以及手性面等的分 类 ${ }^{[12]}$ 进行介绍, 后者则进一步根据原料种类进行综述.

\section{2 去对称化 CuAAC 反应}

利用去对称化策略，从具有潜手性元素的对称双炔 或双叠氮化合物出发, 发展不对称 CuAAC 反应具有下 述特点 ${ }^{[13]}$. 由于反应位点通常远离潜手性中心, 虽然需 要远程控制手性中心的构建, 但受位阻效应的影响不 大, 对于构建季碳手性中心较为有利, 其理论收率可以 达到 $100 \%$; 另外，所得手性三氮唑产物还同时具有炔 基或叠氮基团，可方便进行多种后续衍生化. 当然，如 前所述，如何在实现理想立体选择性控制的同时，抑制 非手性双三氮唑的生成来提高单/双三氮唑的比例 $(\mathrm{M} / \mathrm{D})$, 是实现这类反应的关键. 可喜的是, 通过开发新 
型具有较深的手性口袋的配体，结合反应条件的调控， 已发展了一系列成功的去对称化 $\mathrm{CuAAC}$ 反应, 并用于 含三氮唑结构的(碳或磷)中心手性、轴手性和面手性化 合物的高立体选择性合成.

\section{1 去对称化 CuAAC 构建中心手性}

2013 年，周剑等 ${ }^{[10]}$ 提出利用潜手性双炔体系来发 展不对称催化的 CuAAC 反应(Scheme 5). 基于该课题 组对季碳氧化吲哚构建的兴趣 ${ }^{[14]}$, 作者首先设计了 3,3双炔丙基取代的氧化吲哚 11 来进行反应研究. 幸运的 是在手性 PyBOX 13/CuCl 络合物催化下, 使用 2,5-己二 酮这种特殊溶剂, 能够有效抑制非手性双三氮唑的产生 并有利于提高对映选择性; 进一步通过调节炔烃 11 和 叠氮 12 的物质的量之比, 最终能够以高达 $82 \%$ 的收率 和 $98 \%$ 的 $e e$ 值得到含有炔基和三氮唑基团的全碳季碳 氧化吲哚 14. 这既是首例高对映选择性的不对称 $\mathrm{CuAAC}$ 反应，也是首例成功的基于双炔体系的分子间 去对称化反应 ${ }^{[15]}$. 产物可进行多样性转化得到季碳氧
化吲哚 $18 \sim 21$. 此外, 观察到的明显的负的非线性效应 为 $\mathrm{CuAAC}$ 反应的双核铜催化机理提供了新的佐证.

利用双炔体系的去对称化来发展不对称 $\mathrm{CuAAC}$ 反 应随后得到进一步发展. Stephenson 小组 ${ }^{[16]}$ 尝试了 $\alpha$-双 炔基取代的氰基乙酸甲酯类化合物 22 与苄基叠氮 $12 \mathrm{a}$ 的反应(Scheme 6). 作者篮选了 BOX 配体 26、PHOX 类 单膦配体 27 和 BINAP 等双膦配体，发现采用手性双膦 配体 23 与 $\mathrm{CuI}$ 形成的配合物能以 $6 \%$ 的转化率和 $18 \%$ 的 $e e$ 值到目标化合物 24.

2015 年, 徐利文小组 ${ }^{[17]}$ 设计合成了一种新型的大 位阻多齿手性膦配体 Tao-PHOS 31. 相较于 BINOL 衍生 的 34 和 PyBOX 13 等手性配体, Tao-PHOS 与 $\mathrm{CuF}_{2}$ 形成 的络合物作为催化剂，在基于马来酰胺骨架的双炔 29 与叠氮化合物 30 的 $\mathrm{CuAAC}$ 反应中, 以 $60 \% \sim 80 \%$ 的产 率和 70\% 99\% ee 值得到手性三氮唑 32 (Scheme 7). 这 个反应的特点在于所有反应例子的单/双三氮唑比例均 大于 $12 / 1$, 只有痕量非手性双三氮唑生成. 这说明具有

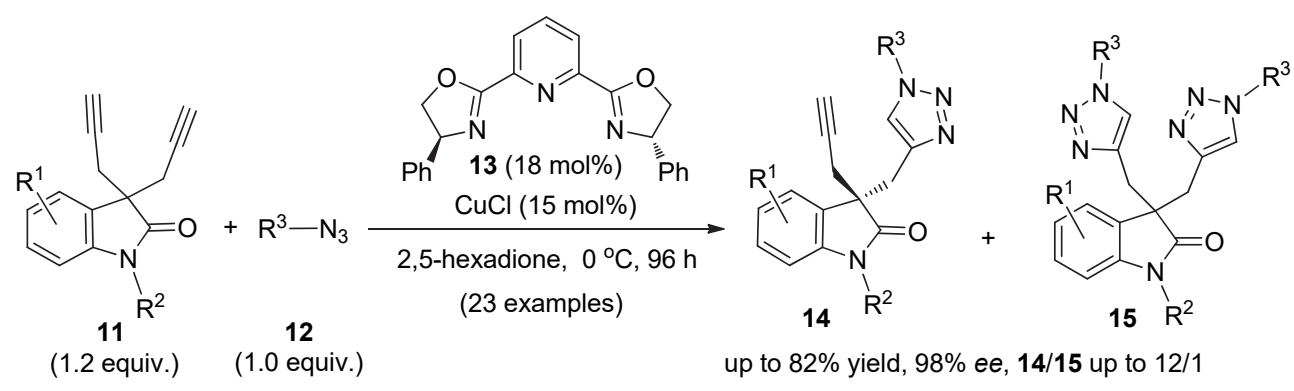

Solvent effects (11a: $R^{1}=H, R^{2}=M e ; 12 a R^{3}=B n, 13 / C u C l=1.2 / 1,10$ mol\% Cat.)

$\begin{array}{cccc}\mathrm{CH}_{2} \mathrm{Cl}_{2} & \text { acetone } & \text { 3-pentone } & \text { 2,5-hexadione } \\ 67 \% \text { ee, } 11 \% \text { yield } & 75 \% \text { ee, } 20 \% \text { yield } & 84 \% \text { ee, } 21 \% \text { yield } & 90 \% \text { ee, } 32 \% \text { yield } \\ \mathbf{1 4 / 1 5}=1 / 4 & \mathbf{1 4 / 1 5}=1 / 2 & \mathbf{1 4 / 1 5}=1 / 2 & 14 / 15=1 / 1\end{array}$<smiles>O=C1Cc2ccccc2C1(Cc1ccccc1)Cc1cn(Cc2ccccc2)nn1</smiles>

18

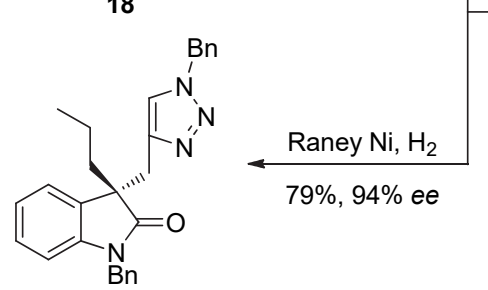

20
Synthetic application

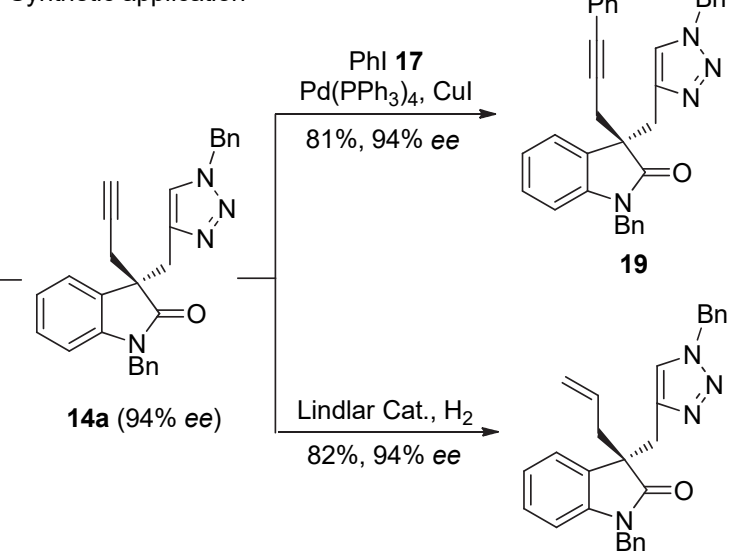

21

图式 5 双炔基取代氧化吲哚的去对称化 $\mathrm{CuAAC}$ 反应

Scheme 5 Desymmetric CuAAC of oxindole-based dialkynes 


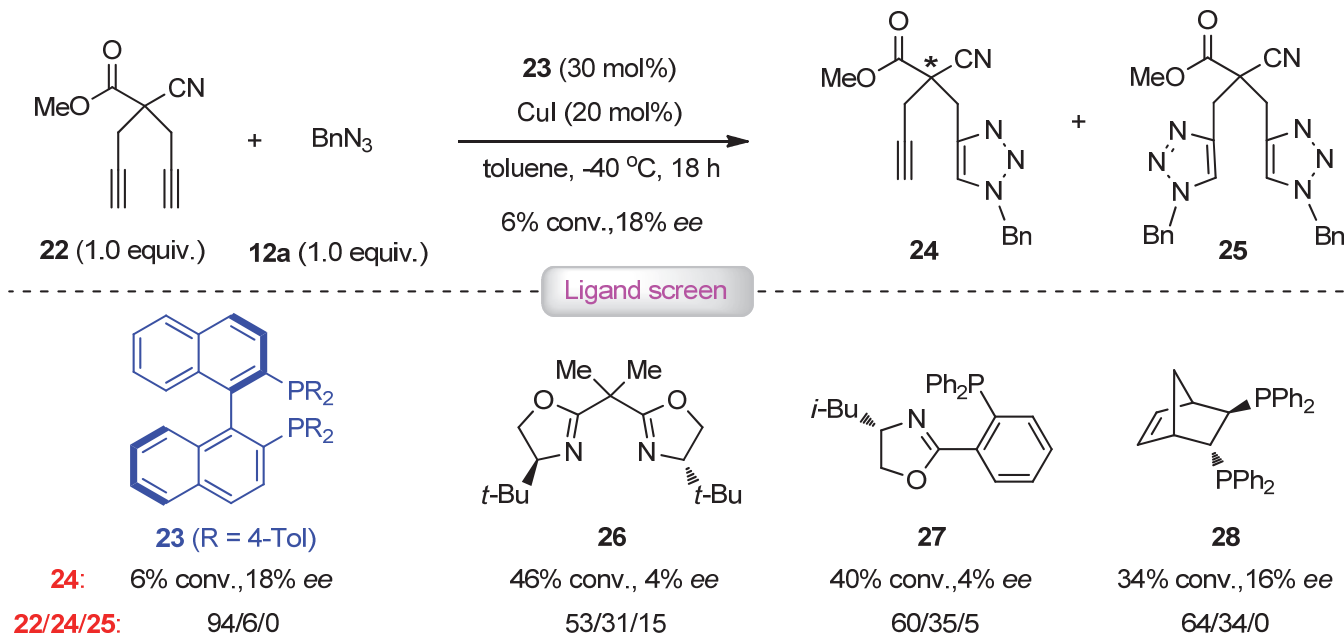

图式 6 氰基乙酸甲酯类双炔的去对称化 CuAAC 反应

Scheme 6 Desymmetric CuAAC of methyl cyanoacetate-based dialkynes

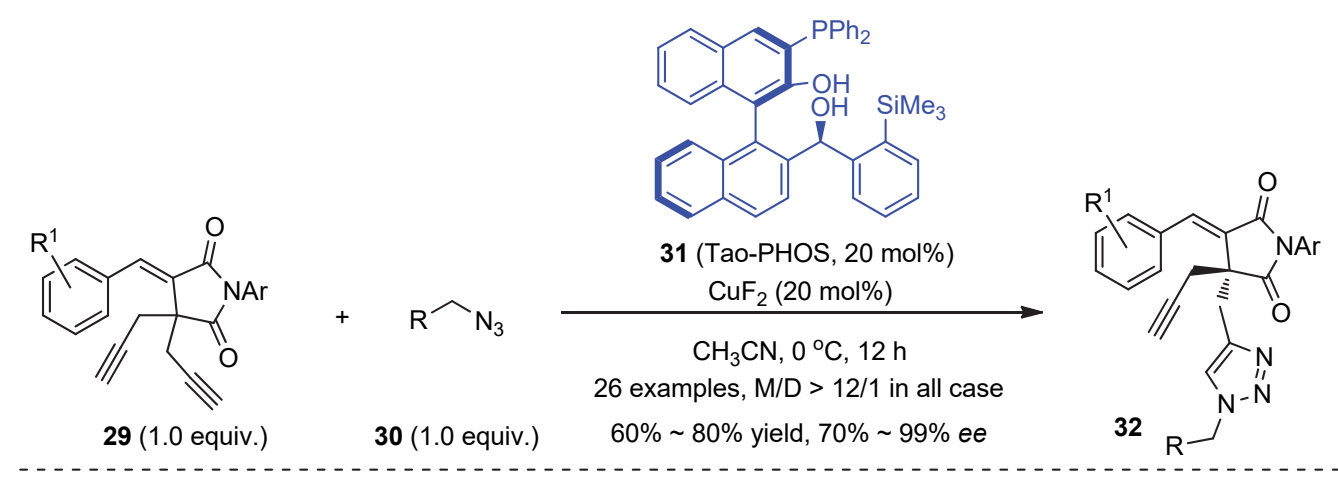

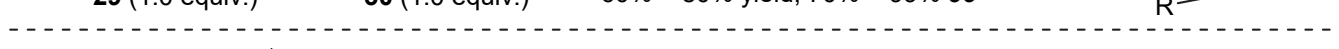

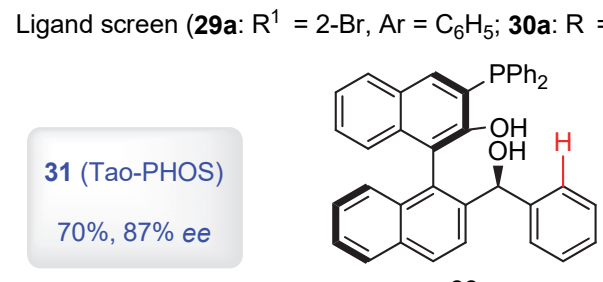

33

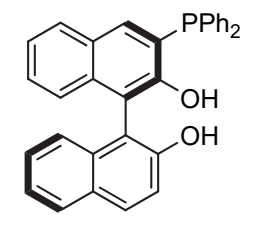

34

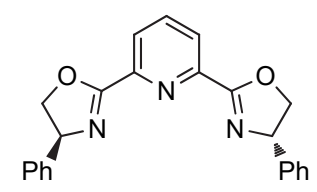

13

$60 \%, 46 \%$ ee

$69 \%, 0 \%$ ee $<10 \%, 23 \%$ ee

Control experiments (29b: $\left.\mathrm{R}^{1}=2-\mathrm{F}, \mathrm{Ar}=4-\mathrm{EtOC}_{6} \mathrm{H}_{4} ; \mathbf{3 0 b}: \mathrm{R}=4-\mathrm{MeC}_{6} \mathrm{H}_{4}\right)$

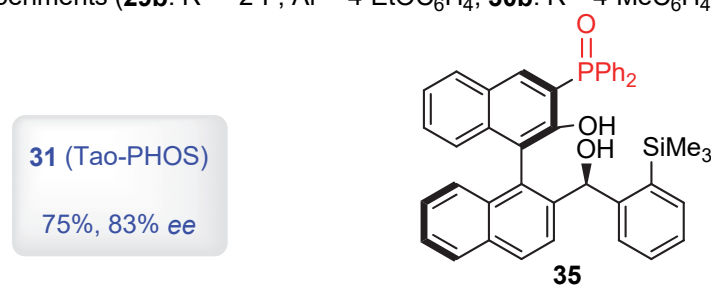

$45 \%, 0 \%$ ee

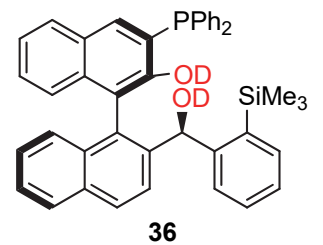

trace product

图式 7 基于马来酰胺类双炔的去对称化 $\mathrm{CuAAC}$ 反应

Scheme 7 Desymmetric CuAAC of maleimide-based dialkynes

手性深腔的新型配体, 有望抑制生成双三氮唑的过度反 应. 但这类配体的手性控制的能力还需进一步改善, 因 为在所报道的 26 个例子中, 仅两例能取得大于 $90 \%$ 的 $e e$ 值, 进一步研究表明 Tao-PHOS 配体中的叔膦、羟基
和三甲基硅基(TMS)对反应的活性和立体选择性均有着 至关重要的影响 ${ }^{[18]}$, 当配体上的叔膦换成膦氧基团时, 反应的 $e e$ 值为零; 而当羟基上的氢原子被氞替换时，只 能得到痕量产物; 使用不含 TMS 的配体 $\mathbf{3 3}$ 时，反应仅 
取得 $46 \%$ ee. 因此, 作者认为配体通过叔膦与铜配位, 羟基与双炔 29 的羰基存在氢键作用, 而大位阻 TMS 的 存在有利于提高反应的手性控制. 此外, 非线性效应和 质谱研究结果也支持反应经历了双核铜催化的机理.

2018 年, 他们 ${ }^{[19]}$ 进一步使用 Tao-PHOS 配体 31 与 纳米 $\mathrm{Cu}_{2} \mathrm{O}$ 形成的手性催化剂, 对吡唑啉酮衍生的双炔 37 与叠氮 30 的去对称化 CuAAC 反应进行了研究 (Scheme 8), 最终以高达 $70 \%$ 的产率和 $90 \%$ 的 $e e$ 值得到 了目标化合物 38, 单/双三氮唑比例均大于 20/1. 双核铜 催化的机理再次得到非线性效应和质谱研究的支持. 值 得一提的是, 该工作是首例非均相的不对称 $\mathrm{CuAAC}$ 反 应，手性催化剂能够重新回收利用.

具有磷手性中心的光学活性膦氧化合物, 在医药、 农药和手性催化剂等领域应用很广 ${ }^{[20]}$, 其不对称催化 合成近年来受到了广泛关注 ${ }^{[21]}$, 但可用于模块化合成 磷手性砌块的方法依然很少. 最近, 周剑小组 ${ }^{[22]}$ 实现了

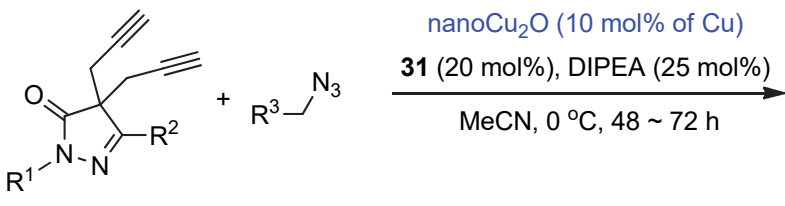

37 (1.0 equiv.) $\quad 30$ (1.1 equiv.)

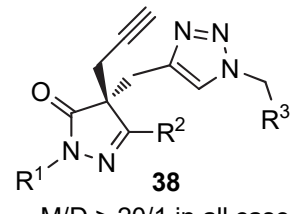

32 examples

$51 \% \sim 70 \%$

$68 \% \sim 90 \%$ ee

$M / D>20 / 1$ in all case

图式 8 吡唑啉酮类双炔的去对称化 $\mathrm{CuAAC}$ 反应

Scheme 8 Desymmetric CuAAC of pyrazolone-derived bisalkynes

潜手性双炔基膦氧化合物的不对称 $\mathrm{CuAAC}$ 反应，高对 映选择性地合成了乙炔基取代的磷手性膦氧化合物 (Scheme 9). 作者设计合成的吡定 C-4 位带有大位阻柔

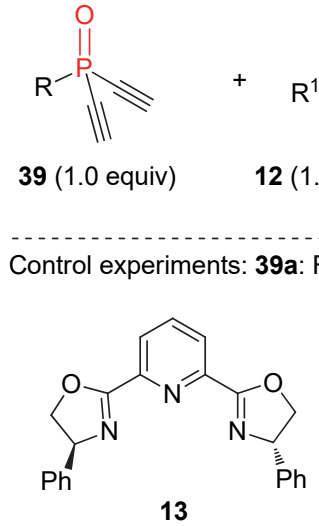

$63 \%, 83 \%$ ee

$\mathbf{4 1 / 4 2}=6.8: 1$

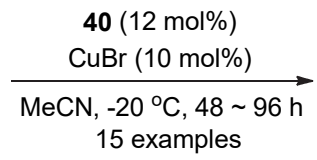

$51 \% \sim 85 \%, 75 \% \sim 96 \%$ ee

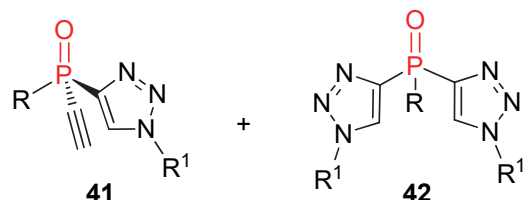

41 2b: $\mathrm{R}^{1}=4-\mathrm{MeC}_{6} \mathrm{H}_{4} \mathrm{CH}_{2}$ with $\mathrm{CuC}$<smiles>c1ccc(COc2cc(C3=N[C@@H](c4ccccc4)CO3)nc(C3=N[C@H](c4ccccc4)CO3)c2)cc1</smiles>

$71 \%, 90 \%$ ee

$\mathbf{4 1 / 4 2}=10.4: 1$

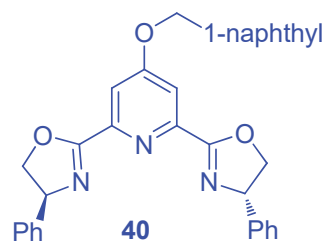

$80 \%, 93 \%$ ee

$\mathbf{4 1 / 4 2}=13.9 \cdot 1$

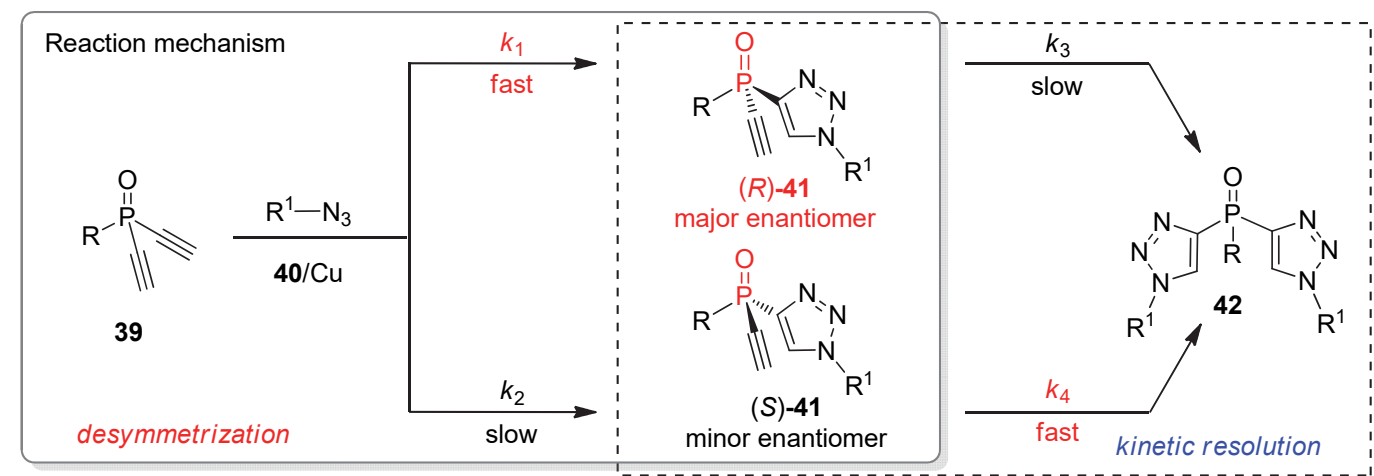

Synthetic application<smiles>[R1]n1cc(P(=O)(c2ccccc2)c2cccc3ccccc23)nn1</smiles><smiles>[R]n1cc(P(#Cc2ccccc2)c2cccc3ccccc23)nn1</smiles>

$44 a, 65 \%$ for 2 steps
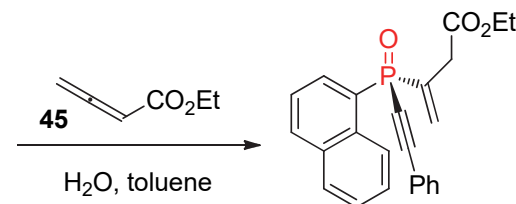

46a, $85 \%, 95 \%$ ee

图式 9 基于膦氧双炔的去对称化 $\mathrm{CuAAC}$ 反应

Scheme 9 Desymmetric CuAAC of phosphole-based dialkynes 
性基团的 PyBOX 配体 $\mathbf{4 0}$ 与 $\mathrm{CuBr}$ 形成的络合物, 能顺 利催化双乙炔基膦氧 39 与叠氮 12 的反应，以高达 $85 \%$ 的产率和 $96 \%$ 的 $e e$ 值得到目标产物 41 .

相较于母体 PyBOX 配体 13, 吡定环 C-4 位大位阻 取代基的引入可以有效提高对映选择性, 并显著抑制非 手性双三氮唑的生成, 使得单/双三氮唑比例最高能达 23/1. 根据可能的双核铜催化机理, 作者认为吡啶环的 C-4 位大位阻基团有助于催化剂形成更好的手性口袋, 不但提高反应的立体选择性, 而且使得潜手性双炔原料 39 的炔基比起位阻更大的手性产物 41 的炔基, 更容易 被催化剂识别与活化，从而抑制双三氮唑的生成.

进一步对反应的单/双三氮唑的比值和产物 $e e$ 值随 时间的变化进行监测, 发现反应涉及去对称化和动力学 拆分的协同过程. 在去对称化过程中, 形成 $R$ 构型产物 的反应速度快于生成 $S$ 构型的速度 $\left(k_{1}>k_{2}\right)$; 与此同时, $R$ 构型的手性单三氮唑产物会通过动力学拆分, 进一步 提高自身的 $e e$ 值, 但是反应的单/双三氮唑比例也逐渐 降低. 而新发展的手性配体在上述两个过程中均能取得 更为优秀的立体选择性. 此外, 所得手性产物中同时含 乙炔基和三氮唑基团，能够作为手性膦的合成子，进一 步转化为手性叔膦 44a 和手性膦氧 46a (Scheme 9).

利用这一方法进一步实现了手性膦杂茂化合物的 合成 ${ }^{[23]}$. 膦杂茂化合物在有机光电材料研究中具有重 要的应用价值, 然而尚无手性合成方法. C-4 位含有大 位阻芳基取代的 PyBOX 配体 48/CuBr 配合物催化剂能 顺利催化二苯并膦杂茂二炔 47 与叠氮 12 的不对称 $\mathrm{CuAAC}$ 反应, 以 $65 \% \sim 81 \%$ 的产率和 $92 \% \sim 99 \%$ ee 值
得到结构新颖的磷手性膦杂茂化合物 49 (Scheme 10), 双三氮唑副产物可以明显得到抑制，49/50 的比例最高 达到 18/1. 对所得手性膦杂茂衍生物的光电性质进行研 究发现，其紫外吸收和苂光发射光谱可以很容易得到调 节。

上述反应中，二苯并膦杂茂二炔 47 中的乙炔基反 应位点与潜手性磷原子相隔四根共价键，这在远程控制 实现的高对映选择性去对称化反应中很少见. 而 PyBOX 配体 C-4 位大位阻基团在远程控制立体选择性 和抑制非手性双三氮唑形成方面再次展示了明显的作 用. 基于这一点, 周剑课题组 ${ }^{[24]}$ 进一步利用这类配体成 功实现了高对映选择性的潜手性双叠氮化合物的去对 称化 $\mathrm{CuAAC}$ 反应. 手性 $\mathrm{PyBOX}$ 配体 54 与 $\mathrm{CuCl}$ 形成的 配合物催化剂, 顺利地催化双叠氮取代的叔醇 51 与末 端炔烃 52 的反应，取得中等的收率以及中等到优秀的 对映选择性. 进一步研究发现，对于碘代的非末端炔烃 53, 反应同样可以顺利进行，以高达 $68 \%$ 的收率和 $97 \%$ 的 $e e$ 值得到含碘取代的三氮唑基团的 $\beta$-叠氮手性叔醇 56 (Scheme 11), 这也是首例不对称铜催化的叠氮和碘 代炔烃的环加成反应. 通过对叠氮或羟基的转化，所得 手性叠氮醇 55a 能够顺利衍生为手性叔醇 57 59 或啞 唑啉硫酮 61. 该反应同样涉及去对称化和动力学拆分 的协同作用，产物的 ee 值随反应进行逐渐升高，而单/ 双三氮唑的比例随之下降. 尽管两类反应中均能取得良 好到优秀的对映选择性, 但是单双比最高仅为 3/1. 这 进一步说明发展去对称化 $\mathrm{CuAAC}$ 反应还需在配体设计 方面继续努力.

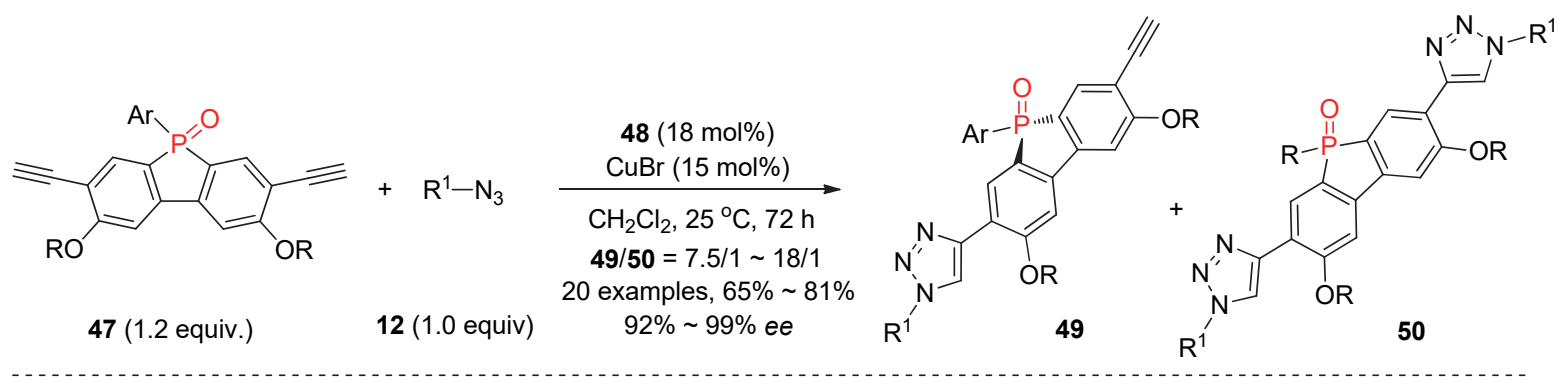

Control experiments [47a: $\mathrm{Ar}=4-\mathrm{MeC}_{6} \mathrm{H}_{4}, \mathrm{R}={ }^{n} \mathrm{Pr} ;$ 12c: $\mathrm{R}^{1}=\mathrm{PhthN}\left(\mathrm{CH}_{2}\right)_{4}$ with $\left.\mathrm{CuCl}\right]$

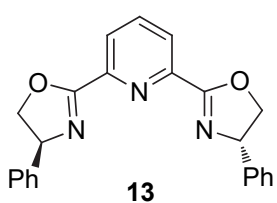

$45 \%, 88 \%$ ee $\mathbf{4 9} / \mathbf{5 0}=1.9 / 1$<smiles>O=[Ge]C1COC(c2cc(OCc3cccc4ccccc34)cc(C3=NC(c4ccccc4)CO3)n2)=N1</smiles>

$57 \%, 93 \%$ ee $\mathbf{4 9} / \mathbf{5 0}=3.5 / 1$

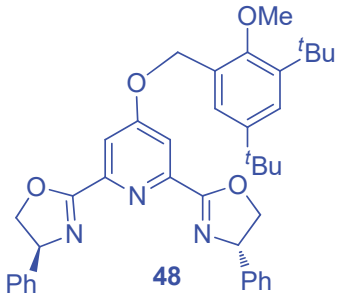

$62 \%, 96 \%$ ee

$\mathbf{4 9} / \mathbf{5 0}=4.6 / 1$

图式 10 基于膦杂茂二炔的去对称化 $\mathrm{CuAAC}$ 反应

Scheme 10 Desymmetric CuAAC of phosphole-based dialkynes 


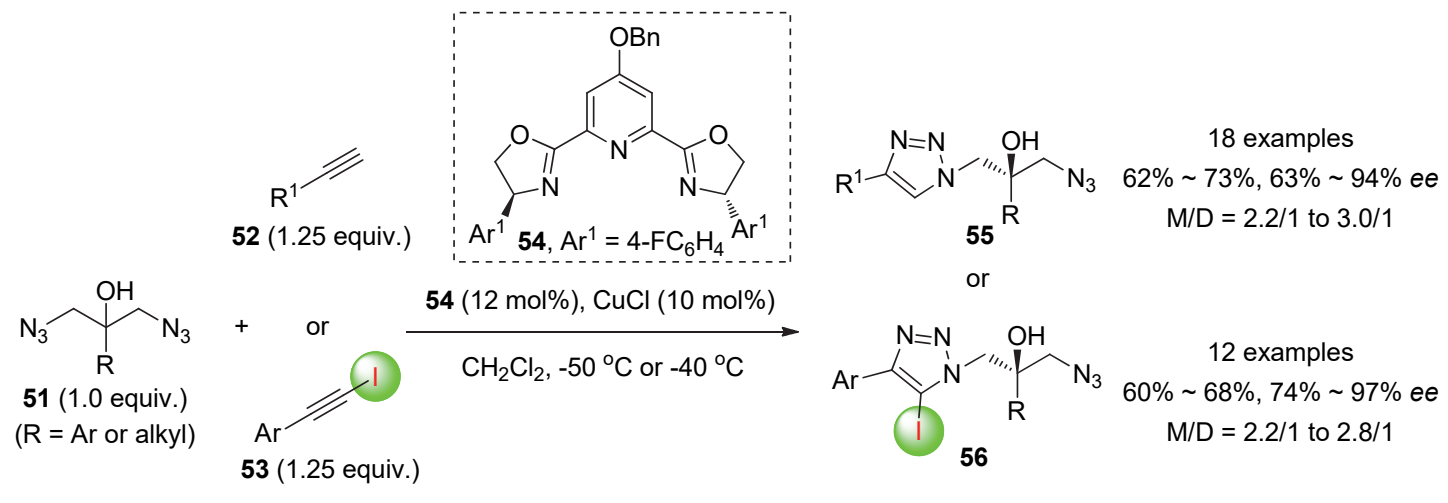

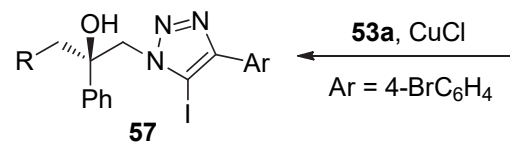

$99 \%, 99 \%$ ee

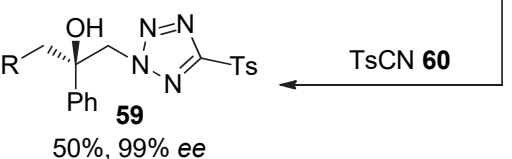

Synthetic application

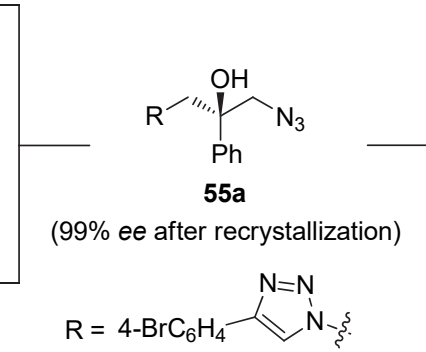

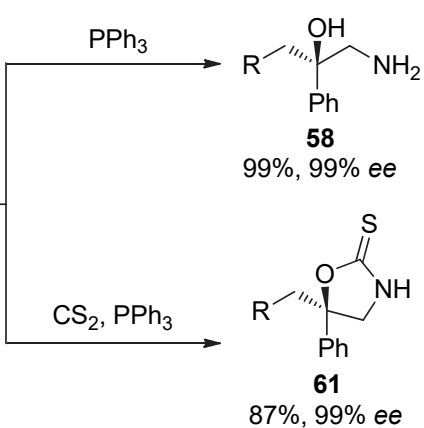

图式 11 基于双叠氮醇的去对称化 $\mathrm{CuAAC}$ 反应

Scheme 11 Desymmetric CuAAC of prochiral diazido alcohols

\section{3 去对称化 CuAAC 构建轴手性}

轴手性存在于许多生物活性分子以及手性配体和 催化剂中, 发展构建轴手性的新方法是不对称催化的研 究热点 ${ }^{[25]} .2014$ 年, Uozumi 等 ${ }^{[26]}$ 首次通过去对称化的 CuAAC 反应合成了轴手性的联芳基化合物(Scheme 12). 考察各类配体后发现 $\mathrm{PyBOX}$ 配体 $\mathbf{6 3}$ 和 $(\mathrm{CuOTf})_{2} \cdot \mathrm{C}_{6} \mathrm{H}_{6}$ 生成的络合物催化剂, 在潜手性联芳基双炔 62 与苄基 叠氮 12a 的反应中, 以最高达 $76 \%$ 的产率和 $99 \%$ 的 $e e$ 值得到了含三氮唑结构的轴手性联二芳基化合物 64 . 反应的单/双三氮唑的比例并不理想, 最高只有 3.8/1. 作者还发现当芐基叠氮的用量由 1 equiv. 增加到 2 equiv. 时, 产物的 $e e$ 值从 $80 \%$ 上升到 $97 \%$, 而单双比则由 $6 / 1$ 下降到 1/2.8. 据此, 作者首次发现在不对称去对称化的 $\mathrm{CuAAC}$ 反应中, 对映选择性的控制可能是去对称化和 动力学拆分协同作用的结果 ${ }^{[27]}$.

\section{4 去对称化 CuAAC 构建面手性}

不对称 $\mathrm{CuAAC}$ 反应也可以用于构建平面手性 ${ }^{[28]}$. 2019 年, Stephenson 等 ${ }^{[29]}$ 报道了二茂铁衍生的双炔化合 物 69 与苠基叠氮 12a 的去对称 CuAAC 反应(Scheme 13). 他们发现在如 Scheme 13 所示的反应条件下, PyBOX 70、BOX 66 和面手性双膦配体 73 均能够取得优 异的对映选择性, 而使用双膦配体 73 所得产物的绝对 构型截然相反. 此外, 二茂铁双炔底物中的羟基对反应
的活性和立体选择性有至关重要的影响，将羟基改为酯 基时，目标产物的产率和 $e e$ 值均大幅度下降. 作者也发 现该反应是去对称化和动力学拆分协同进行的过程. 尽 管该反应中只有一个底物能取得优秀的立体选择性，且 伴随几乎等物质的量双三氮唑副产物的生成, 但是它显 示不对称 $\mathrm{CuAAC}$ 反应可以为炔基或叠氮基取代的具有 面手性的化合物的构建提供了一种新方法.

\section{3 动力学拆分 CuAAC 反应}

基于外消旋叠氮或炔烃的(动态)动力学拆分是实现 不对称催化 CuAAC 反应的另一重要途径, 这两种方法 各有其特点 ${ }^{[30]}$. 理想的动力学拆分能通过一步反应同 时得到两种手性化合物：含手性的三氮唑以及含手性的 炔烃(或叠氮)，虽然回收率和产率最高各只有 $50 \%$, 但 是对于多样性合成手性化合物有帮助; 而动态动力学拆 分反应则能够以 $100 \%$ 的理论产率得到含手性的三氮唑 化合物.

除了 Fokin 等 ${ }^{[8]}$ 的早期探索外, Fossey 等 ${ }^{[31]}$ 在 2015 年利用周剑小组所发展的反应条件，尝试了对外消旋 3炔丙基氧化吲哚 74 的动力学拆分(Scheme 14a), 但是结 果并不理想. 当转化率在 $50 \%$ 时, 原料 $\mathbf{7 5}$ 能以 $81 \%$ ee 值回收; 而转化率在仅为 $8 \%$ 时，产物 76 才能取得 $80 \%$ 的 $e e$ 值. 随后, 他们使用 BINOL 衍生的硼酸酯 80 作为 

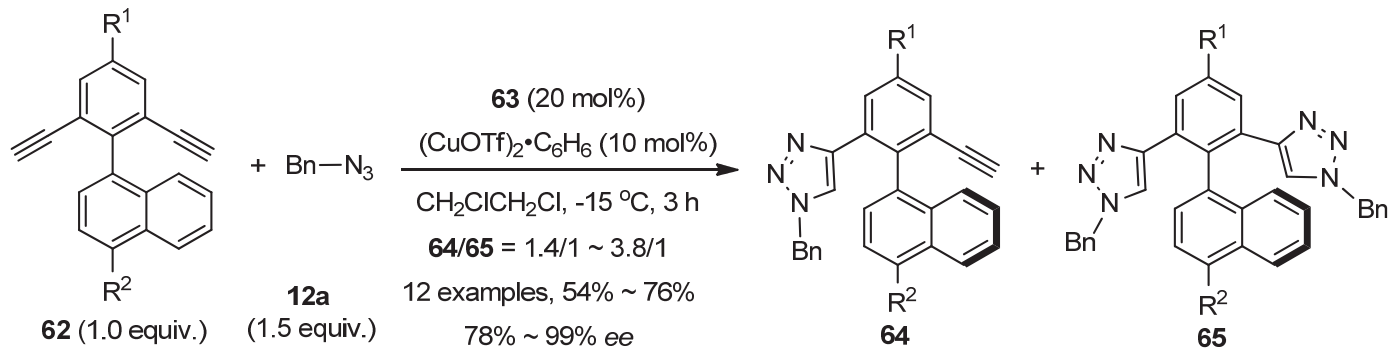

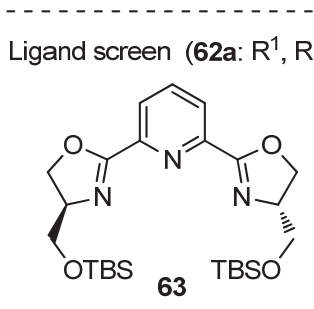

$67 \%, 79 \%$ ee

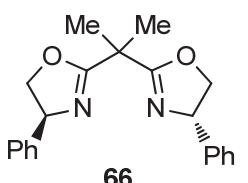

$34 \%, 10 \%$ ee

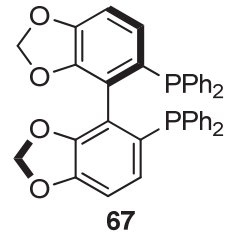

$62 \%, 3 \%$ ee

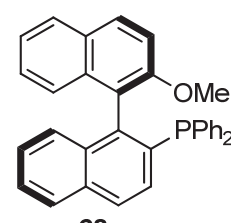

68

图式 12 基于联芳基双炔的去对称化 $\mathrm{CuAAC}$ 反应

Scheme 12 Desymmetric CuAAC of biaryls-derived dialkynes
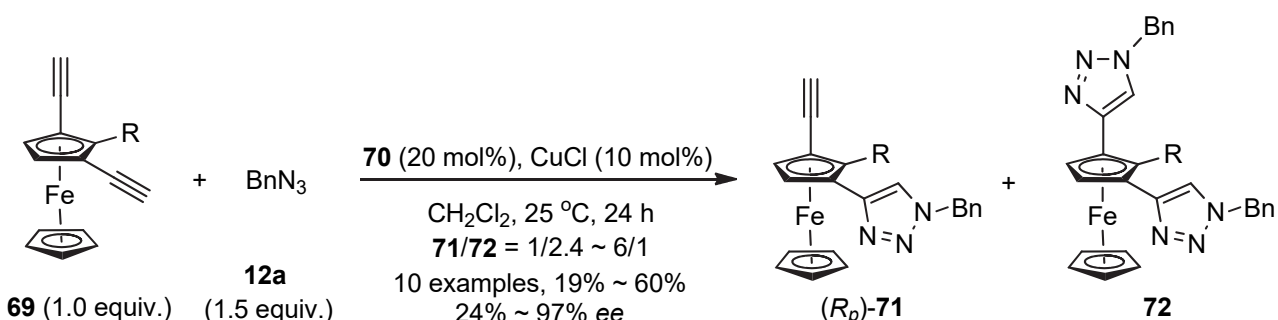

69 (1.0 equiv.) (1.5 equiv.) $24 \% \sim 97 \%$ ee

$\left(R_{p}\right)-71$

72

Substrate screen (with $\mathbf{7 0}$ as ligand)

$\begin{array}{cccccc}\mathrm{R} & \mathrm{CH}_{2} \mathrm{OH} & \mathrm{CH}_{2} \mathrm{OMe} & \mathrm{CH}_{2} \mathrm{OBn} & \mathrm{CO}_{2} \mathrm{Me} & \mathrm{CO}_{2} \mathrm{Bn} \\ \text { 71:72:69 } & 42: 49:-- & 47: 49:-- & 51: 32: 8 & 51: 30: 17 & 34: 47: 1 \\ \text { ee of } \mathbf{7 1} & 97 \% & 52 \% & 42 \% & 31 \% & 38 \%\end{array}$

Ligand screen $\left(\mathrm{R}=\mathrm{CH}_{2} \mathrm{OH}\right)$

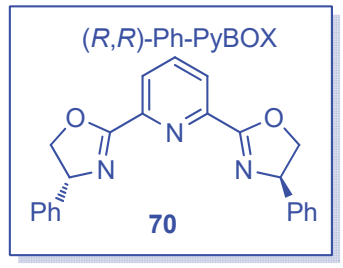

$42 \%$ yield, $97 \%$ ee $71 / 72=42 / 49$

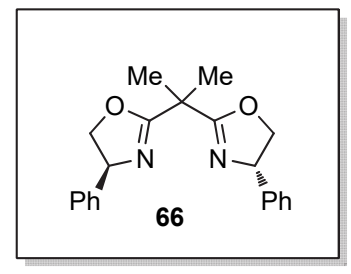

$11 \%$ yield, $>99.5 \%$ ee

$\mathbf{7 1 / 7 2}=11 / 67$

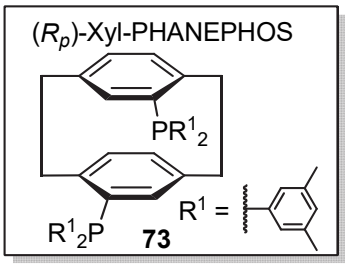

$32 \%$ yield, $97 \%$ ee

$71 / 72=32 / 49$

图式 13 基于二茂铁双炔的去对称化 CuAAC 反应

Scheme 13 Desymmetric CuAAC of bisalkynylferrocenes

手性辅基, 研究了手性底物诱导的动力学拆分, 但是选 择性因子最高也仅为 4.1 (Scheme 14b) ${ }^{[32]}$. 他们还尝试 了外消旋炔烃 74 和外消旋叠氮 1 的不对称 $\mathrm{CuAAC}$ 反应. 当转化率为 $36 \%$ 时, 能以 $84: 16$ 的 $d r$ 值及 $90 \%$ 的 $e e$ 值得到目标化合物 $\mathbf{8 5}$, 显示能通过不对称 $\mathrm{CuAAC}$ 反应 构建两个手性中心(Scheme 14c) ${ }^{[33]}$.
2019 年 Topczewski 等 ${ }^{[34]}$ 使用 $1.25 \mathrm{~mol} \%$ 的 PyBOX $88 / \mathrm{Cu}(\mathrm{I})$ 络合物为催化剂, 在外消旋二级叠氮 86 与末端 炔烃 87 的不对称 CuAAC 反应中，能以高达 $97 \%$ 的产率 和 94\% ee 值得到含手性的三氮唑 89 (Scheme 15). 具有 环状结构的外消旋叠氮化合物的反应结果优于非环状 的, 然而回收原料的 $e e$ 值仍不理想. 
(a)<smiles>C#CCC1(C)C(=O)Nc2ccccc21</smiles>

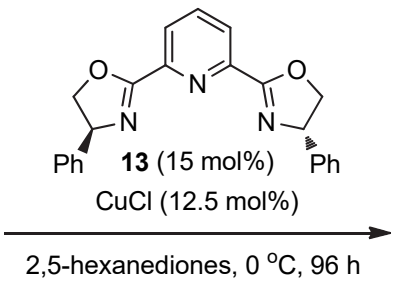

$s=22.1 \pm 0.5$<smiles>C#CC[C@]1(C)C(=O)Nc2ccccc21</smiles>

75<smiles>C[C@]1(Cc2cn(Cc3ccccc3)nn2)C(=O)Nc2ccccc21</smiles>

76

74 (1.0 equiv.) $\quad$ 12a ( 0.6 equiv.) $50 \%$ conv., $81 \%$ ee

$8 \%$ conv., $80 \%$ ee

(b)<smiles>C#CC(N)c1ccccc1</smiles>

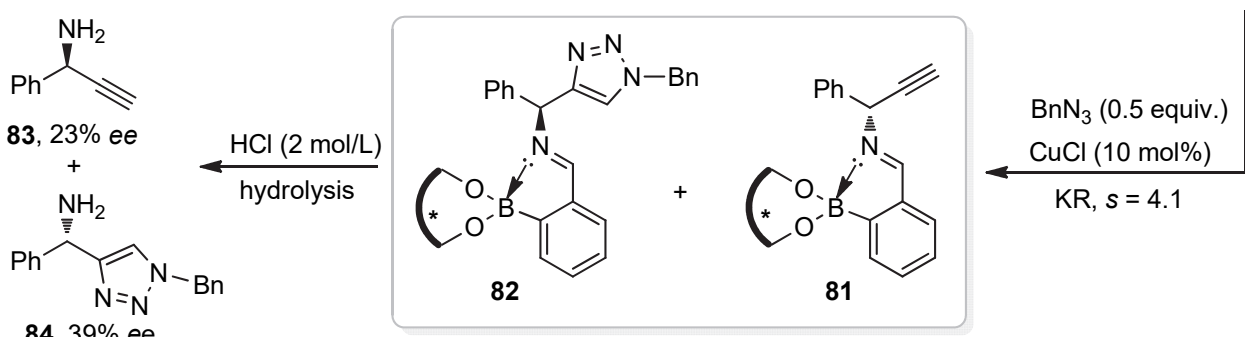

$\mathbf{8 4}, 39 \%$ ee

(c)

(c)

74 (1.0 equiv.) 1 (1.0 equiv.)

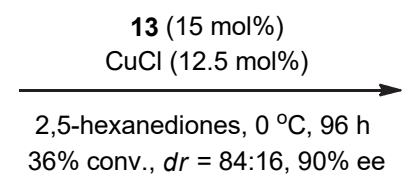

$36 \%$ conv., $d r=84: 16,90 \%$ ee

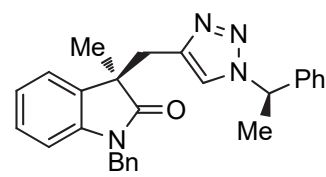

85

图式 14 外消旋氧化吲哚类炔烃的动力学拆分 $\mathrm{CuAAC}$ 反应

Scheme 14 CuAAC based on kinetic resolutions of oxindole-based racemic alkynes<smiles>[Y]CC([NH3+])c1c([R])cccc1[X]</smiles>

$+{ }_{\mathrm{R}} \geqslant$

87 (1.0 equiv.)

86 (2.5 equiv.)<smiles>[Y]C1COC(c2cccc(C3=N[C@@H]([AlH2])CO3)n2)=N1</smiles>
$(\mathrm{CuOTf})_{2} \cdot \mathrm{PhMe}(1.25 \mathrm{~mol} \%)$

$\mathrm{PhCF}_{3},-15^{\circ} \mathrm{C}, 72 \mathrm{~h}$

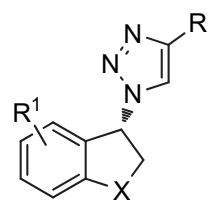

89

30 examples $37 \% \sim 97 \%$ yield, $58 \% \sim 94 \%$ ee $\left(\mathrm{Ar}=4-\mathrm{FC}_{6} \mathrm{H}_{4}\right)$

Selected examples $\left(\mathrm{R}=4-\mathrm{MeC}_{6} \mathrm{H}_{4}\right)$<smiles>[R]c1cn([C@@H]2CCOc3ccccc32)nn1</smiles>

$89 a$<smiles>Pc1cn([C@H]2CCNc3ccccc32)nn1</smiles>

$89 b$

$90 \%, 80 \%$ ee $\quad 96 \%, 62 \%$ ee<smiles>Pc1cn([C@@H]2CCc3ccccc32)nn1</smiles>

$89 \mathrm{c}$

$97 \%, 88 \%$ ee

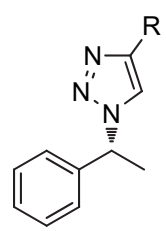

89d $37 \%, 58 \%$ ee
Gram-scale reaction

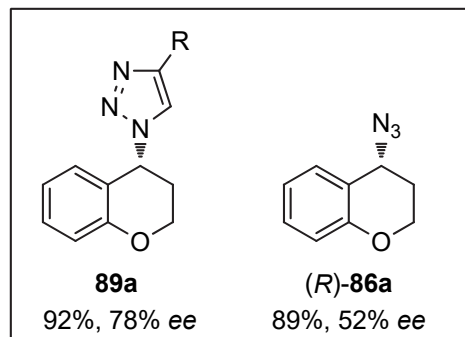

图式 15 外消旋二级叠氮的动力学拆分 $\mathrm{CuAAC}$ 反应

Scheme 15 CuAAC based on kinetic resolutions of racemic secondary azides 
例如, 作者尝试了克级规模的反应, 能以 $92 \%$ 的产 率和 $78 \%$ 的 $e e$ 值制备 $1.2 \mathrm{~g}$ 含手性的三氮唑 $89 \mathrm{a}$, 同时 以 $52 \%$ ee 值回收手性叠氮 86a. 需要指出的是该方法的 立体选择性仍有很大的提升空间, 在所报道的 30 个例 子中，仅有两例的对映选择性超过 $90 \%$.

在基于膦氧双炔的去对称化 $\mathrm{CuAAC}$ 反应的基础上, 周剑小组进一步发展了对外消旋乙炔基膦氧的动力学 拆分 $\mathrm{CuAAC}$ 反应, 通过简单的改变吡啶环 C-4 位含大 位阻的 PyBOX 型配体的取代基, 使用配体 54 或 92 与 $\mathrm{CuBr}$ 的络合物催化剂, 可以高效地催化外消旋单乙炔 基膦氧 90 与叠氮 12 的 $\mathrm{CuAAC}$ 反应 ${ }^{[22]}$, 以良好到优秀 的对映选择性分别得到具有磷手性的单乙炔基膦氧 $(S)-91$ 和三氮唑膦氧 $(R)$-93 (Scheme 16). 所得手性乙炔 基膦氧 91b 经 Sonogashira 偶联、 $\mathrm{HSiCl}_{3}$ 还原, 即可得到 手性保持的磷手性叔膦 94b, 其作为亲核催化剂可顺利 地催化烯酮 95 和联烯酸酯 96 的不对称环加成反应, 并 取得中等的产率和对映选择性.
高效的动态动力学拆分 ${ }^{[35]}$ 的不对称 $\mathrm{CuAAC}$ 反应能 够把外消旋底物全部转换为单一构型的含手性的三氮 唑化合物，产率最高可达 $100 \%$. 发展合适的方法使外 消旋底物发生消旋化, 且能够与动力学拆分过程相结 合, 是实现动态动力学拆分的关键. 2019 年, Topczewski 等 ${ }^{[36]}$ 在这一领域实现了突破，他们结合烯丙基叠氮的 3,3-Winstein 重排首次实现了动态动力学拆分的 $\mathrm{CuAAC}$ 反应(Scheme 17). 利用 PyBOX 100/Cu(I)络合物催化剂, 通过调节温度来平衡叠氮的消旋化和动力学拆分的反 应速率，最终能够以高达 $99 \%$ 的产率和 $99 \%$ 的对映选择 性得到烯丙基取代的手性三氮唑化合物 101. 研究发现, PyBOX 配体相对其他类型的氮配体或膦配体, 能实现 更优异的立体选择性和催化活性, 催化剂用量仅需 1.25 $\mathrm{mol} \%$. 反应的底物普适性较好, 具有五元、六元、七元 结构的环状叠氮或开链状叠氮, 如 $101 \mathrm{a} \sim 101 \mathrm{e}$, 以及含 有复杂药物分子结构的端炔, 如 101f, 均具有良好的普 适性，充分展现了该方法的实用价值.<smiles>[R]P([R])(=O)C#C</smiles>

(S)-91

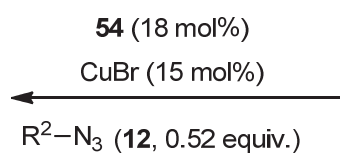

11 examples $42 \% \sim 48 \%$ recovery, $85 \% \sim 99 \%$ ee<smiles>[R]P([R])(=O)C#C</smiles>

$( \pm)-90$

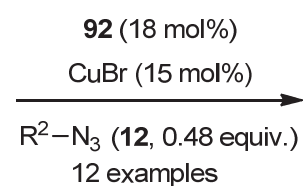

$82 \% \sim 94 \%, 80 \% \sim 93 \%$ ee

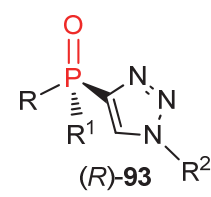

Selected examples $\left(\mathrm{R}^{2}=4-\mathrm{Me}-\mathrm{C}_{6} \mathrm{H}_{4}\right)$

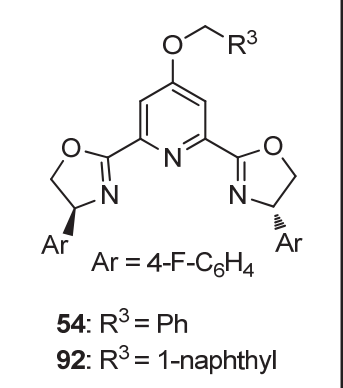

54: $\mathrm{R}^{3}=\mathrm{Ph}$

92: $\mathrm{R}^{3}=1$-naphthy<smiles>C#CP(=O)(c1ccccc1)c1ccccc1</smiles>

91a, $42 \%, 96 \%$ ee<smiles>[R]n1cc(P(=O)(c2ccccc2)c2ccccc2C)nn1</smiles>

93a, $94 \%, 90 \%$ ee

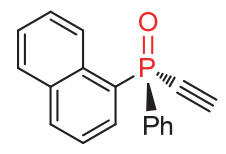

91b, $45 \%, 97 \%$ ee

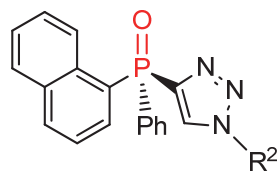

93b, $86 \%, 90 \%$ ee

\section{Synthetic application}<smiles>C#CP(=O)(c1ccccc1)c1cccc2ccccc12</smiles>

91b (96\% ee)<smiles>C=CC(=O)OCC</smiles>

95

96
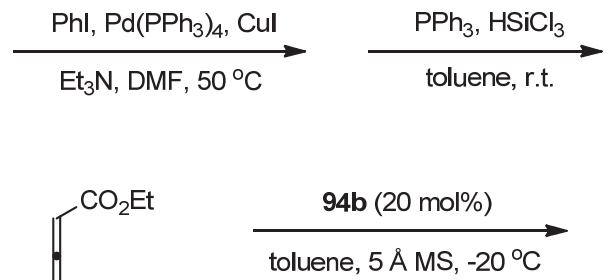

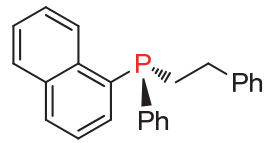

94b, $68 \%, 94 \%$ ee

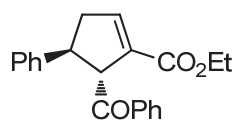

$97,63 \%, 68 \%$ ee

图式 16 外消旋单乙炔基膦氧的动力学拆分 $\mathrm{CuAAC}$ 反应

Scheme 16 CuAAC based on kinetic resolutions of racemic ethynylphosphine oxides 


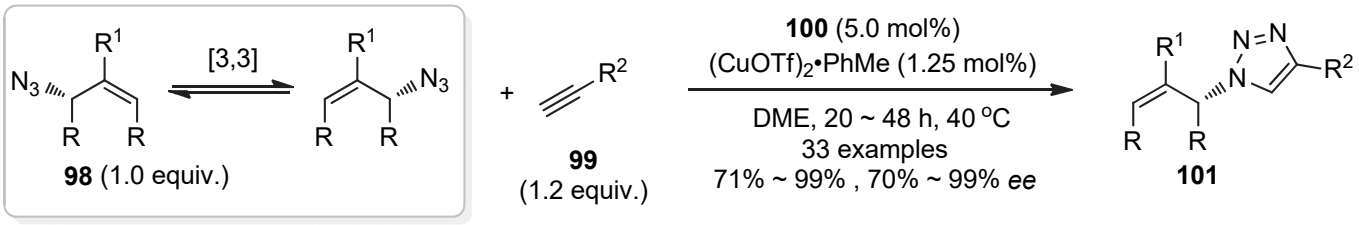

Ligand screen [98a: $\mathrm{R}=\left(\mathrm{CH}_{2}\right)_{3}, \mathrm{R}^{1}=4-\mathrm{MeOC}_{6} \mathrm{H}_{4}, 99 \mathrm{a}: \mathrm{R}^{2}=\mathrm{CO}_{2}{ }^{t} \mathrm{Bu}$ ]

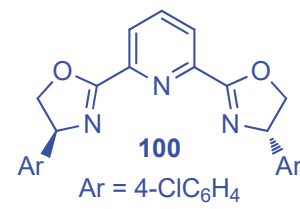

$98 \%, 99 \%$ ee

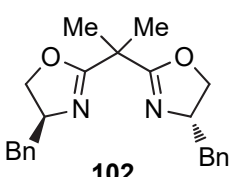

$58 \%, 2 \%$ ee

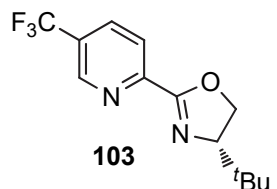

$65 \%, 4 \%$ ee

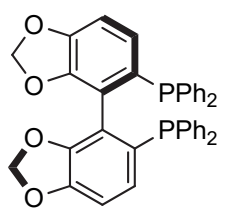

67<smiles>C/C=C(\c1ccccc1)[C@@H](C)n1cc(C(=O)OCC(C)(C)C)nn1</smiles>

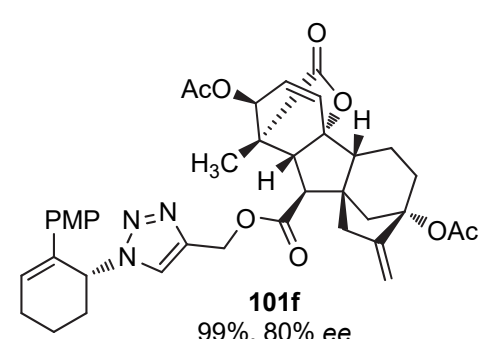

$92 \%, 72 \%$ ee

图式 17 基于动态动力学拆分的 $\mathrm{CuAAC}$ 反应

Scheme 17 CuAAC reaction by dynamic kinetic resolution

\section{4 结论与展望}

综上所述, 过去几年, 不对称 $\mathrm{CuAAC}$ 反应的研究 取得了长足进步. 基于去对称化或(动态)动力学拆分两 种策略，已成功发展出了一系列不对称 $\mathrm{CuAAC}$ 反应， 用于含三氮唑、炔烃或叠氮的碳(磷)中心手性、轴手性 或面手性化合物的高对映选择性合成，有效地拓宽了 CuAAC 反应的应用范围.

尽管如此, 该领域的研究工作仍然有巨大的发展空 间. 首先, 现有方法的底物普适性都不高, 距离将不对 称催化的 $\mathrm{CuAAC}$ 反应发展为多样性和高选择性制备含 叠氮、炔基或三氮唑取代基的手性化合物的目标还很遥 远. 其次, 在发展去对称化的不对称 $\mathrm{CuAAC}$ 反应时, 如 何在取得高对映选择性的同时抑制过度反应生成非手 性双三氮唑依旧是一个难题. 再次, 如何利用不对称 $\mathrm{CuAAC}$ 反应来实现小单元的链接, 合成手性高分子材 料或生物功能材料, 尚待开发. 解决这些问题, 从现有 结果来看, 关键在于发展具有可调手性深腔的新型手性 催化剂. 因此, 通过设计开发新型催化剂来拓展反应的 类型，探索其在有机合成、材料、药物研发等领域的潜 在应用价值, 无疑是这一领域的发展方向. 我们相信不 对称催化的 CuAAC 反应必将迎来更大的发展, 并开拓 出更加广阔的应用前景.

\section{References}

[1] Huisgen, R. Angew. Chem., Int. Ed. 1963, 2, 565.

[2] (a) Tornøe, C. W.; Christensen, C.; Meldal, M. J. Org. Chem. 2002, $67,3057$.

(b) Rostovtsev, V. V.; Green, L. G.; Fokin, V. V.; Sharpless, K. B. Angew. Chem., Int. Ed. 2002, 41, 2596.

[3] Kolb, H. C.; Finn, M. G.; Sharpless, K. B. Angew. Chem., Int. Ed. 2001, 40, 2004.

[4] (a) Meldal, M.; Tornøe, C. W. Chem. Rev. 2008, 108, 2952. (b) Hein, J. E.; Fokin, V. V. Chem. Soc. Rev. 2010, 39, 1302.

[5] (a) Himo, F.; Lovell, T.; Hilgraf, R.; Rostovtsev, V. V.; Noodleman, L.; Sharpless, K. B.; Fokin, V. V. J. Am. Chem. Soc. 2005, 127, 210. (b) Worrell, B. T.; Malik, J. A.; Fokin, V. V. Science 2013, 340, 457.

[6] Kolb, H. C.; Sharpless, K. B. Drug Discovery Today 2003, 8, 1128.

[7] (a) Trost, B. M.; Weiss, A. H. Adv. Synth. Catal. 2009, 351, 963. (b) Ding, P. G.; Hu, X. S.; Zhou, F.; Zhou, J. Org. Chem. Front. 2018, $5,1542$.

[8] Meng, J.-C.; Fokin, V. V.; Finn, M. G. Tetrahedron Lett. 2005, 46, 4543.

[9] Rodionov, V. O.; Fokin, V. V.; Finn, M. G. Angew. Chem., Int. Ed. 2005, 44, 2210.

[10] Zhou, F.; Tan, C.; Tang, J.; Zhang, Y. Y.; Gao, W. M.; Wu, H. H.; Yu, Y. H.; Zhou, J. J. Am. Chem. Soc. 2013, 135, 10994.

[11] Brittain, W. D. G.; Buckley, B. R.; Fossey, J. S. ACS Catal. 2016, 6 , 3629.

[12] Cahn, R. S.; Ingold, C.; Prelog, V. Angew. Chem., Int. Ed. 1966, 5, 385.

[13] Zeng, X.-P.; Cao, Z.-Y.; Wang, Y.-H.; Zhou, F.; Zhou, J. Chem. Rev. 2016, $116,7330$.

[14] Cao, Z.-Y.; Zhou, F.; Zhou, J. Acc. Chem. Res. 2018, 51, 1443.

[15] (a) Wilking, M.; Mück-Lichtenfeld, C.; Daniliuc, C. G.; Hennecke, U. J. Am. Chem. Soc. 2013, 135, 8133.

(b) Mourad, A. K.; Leutzow, J.; Czekelius, C. Angew. Chem., Int. 
Ed. 2012, 51, 11149 .

(c) Sato, Y.; Nishimata, T.; Mori, M. J. Org. Chem. 1994, 59, 6133.

(d) Tanaka, K.; Fu, G. C. J. Am. Chem. Soc. 2002, 124, 10296.

[16] Stephenson, G. R.; Buttress, J. P.; Deschamps, D.; Lancelot, M.; Martin, J. P.; Sheldon, A. I. G.; Alayrac, C.; Gaumont, A.-C.; Page, P. C. B. Synlett 2013, 24, 2723.

[17] Song, T.; Li, L.; Zhou, W.; Zheng, Z.-J.; Deng, Y.; Xu, Z.; Xu, L.-W. Chem.-Eur. J. 2015, 21, 554.

[18] Chen, M.-Y.; Song, T.; Zheng, Z.-J.; Xu, Z.; Cui, Y.-M.; Xu, L.-W. RSC Adv. 2016, 6, 58698.

[19] Chen, M.-Y.; Xu, Z.; Chen, L.; Song, T.; Zhen, Z.-J.; Cao, J.; Cui, Y.-M.; Xu, L.-W. ChemCatChem 2018, 10, 280.

[20] (a) Yao, Q.; Wang, A.; Pu, J.; Tang, Y. Chin. J. Org. Chem. 2014, 34, 292 (in Chinese).

(姚秋丽, 王安俊, 蒲家志, 唐瑜敏, 有机化学, 2014, 34, 292.)

(b) Grabulosa, A.; Granell, J.; Muller, G. Coord. Chem. Rev. 2007, $251,25$.

(c) Wozniak, L. A.; Okruszek, A. Chem. Soc. Rev. 2003, 32, 158.

(d) Pietrusiewicz, K. M.; Zablocka, M. Chem. Rev. 1994, 94, 1375.

[21] (a) Harvey, J. S.; Gouverneur, V. Chem. Commun. 2010, 46, 7477.

(b) Glueck, D. S. Chem.-Eur. J. 2008, 14, 7108.

(c) Glueck, D. S. Synlett 2007, 2627.

[22] Zhu, R.-Y.; Chen, L.; Hu, X.-S.; Zhou, F.; Zhou, J. Chem. Sci. 2020, $11,97$.

[23] (a) Xi, W.; Scott, T. F.; Kloxin, C. J.; Bowman, C. N. Adv. Funct. Mater. 2014, 24, 2572.

(b) Chu, C.; Liu, R. Chem. Soc. Rev. 2011, 40, 2177.

(c) Golas, P. L.; Matyjaszewski, K. Chem. Soc. Rev. 2010, 39, 1338.

[24] Wang, C.; Zhu, R.-Y.; Liao, K.; Zhou, F.; Zhou, J. Org. Lett. 2020, $22,1270$.

[25] (a) Wang, Y.-B.; Tan, B. Acc. Chem. Res. 2018, 51, 534.

(b) Kozlowski, M. C.; Morgan, B. J.; Linton, E. C. Chem. Soc. Rev.
2009, 38, 3193.

(c) Bringmann, G.; Mortimer, A. J. P.; Keller, P. A.; Gresser, M. J.; Garner, J.; Breuning, M. Angew. Chem., Int. Ed. 2005, 44, 5384.

(d) Bringmann, G.; Gulder, T.; Gulder, T. A. M.; Breuning, M. Chem. Rev. 2011, $111,563$.

[26] Osako, T.; Uozumi, Y. Org. Lett. 2014, 16, 5866.

[27] Osako, T.; Uozumi, Y. Synlett 2015, 26, 1475.

[28] (a) Yasue, R.; Kazuhiro, Y. Chem.-Eur. J. 2018, 24, 18575.

(b) Ferbera, B.; Kagan, H. B. Adv. Synth. Catal. 2007, 349, 493.

(c) Dai, L.-X.; Tu, T.; You, S.-L.; Deng, W.-P.; Hou, X.-L. Acc. Chem. Res. 2003, 36, 659 .

[29] Wright, A. J.; Hughes, D. L.; Page, P. C. B.; Stephenson, G. R. Eur. J. Org. Chem. 2019, 7218.

[30] (a) Vedejs, E.; Jure, M. Angew. Chem., Int. Ed. 2005, 44, 3974.

(b) Keith, J. M.; Larrow, J. F.; Jacobsen, E. N. Adv. Synth. Catal. 2001, 343, 5 .

[31] Brittain, W. D. G.; Buckley, B. R.; Fossey, J. S. Chem. Commun. 2015, 51, 17217

[32] Brittain, W. D. G.; Chapin, B. M.; Zhai, W.; Lynch, V. M.; Buckley, B. R.; Anslyn, E. V.; Fossey, J. S. Org. Biomol. Chem. 2016, 14, 10778 .

[33] Brittain, W. D. G.; Dalling, A. G.; Sun, Z.; Duf, C. S. L.; Male, L.; Buckley, B. R.; Fossey, J. S. Sci. Rep. 2019, 9, 15086.

[34] Alexander, J. R.; Ott, A. A.; Liu, E.-C.; Topczewski, J. J. Org. Lett. $\mathbf{2 0 1 9}, 21,4355$.

[35] (a) Bhat, V.; Welin, E. R.; Guo, X.; Stoltz, B. M. Chem. Rev. 2017, 117,4528 .

(b) Pellissier, H. Tetrahedron 2008, 64, 3769.

(c) Huerta, F. F.; Minidis, A. B. E.; Bäckvall, J.-E. Chem. Soc. Rev. 2001, 30, 321.

[36] Liu, E.-C.; Topczewski, J. J. J. Am. Chem. Soc. 2019, 141, 5135.

(Cheng, F.) 